higher response rates have reported similar levels of distress. ${ }^{2}$ A study carried out as part of the NHS workforce survey found no difference in the levels of distress between responders and non-responders, ${ }^{4}$ suggesting that non-response was not systematically related to psychological health. Our results may not be generalisable to non-teaching hospitals and might be confounded by the fact that consultants and house officers were selected from different trusts. In fact, one hospital was a source of both consultants and house officers for this study, and the occupational group differences at this hospital remained significant.

Some of these data are three years old. The continued upheaval in the health service and further reductions in junior house doctors' working hours mean that the differences in psychological distress between senior hospital doctors and their most junior colleagues may now be more pronounced. One approach to addressing this problem could be to tackle specific sources of occupational stress in different groups. For example, our results suggest consultants might benefit from a reduction in their workload, while house officers might benefit if they had greater autonomy.
We would like to thank the consultants and house officers.

Contributors: NK coordinated and collected data for the house officer study, helped analyse the data, and wrote the initial draft of the paper. $\mathrm{CB}$ coordinated the consultant study and commented on drafts of the paper. CS helped to extract and analyse the data, and commented on drafts of the paper. NK and $\mathrm{CB}$ will act as guarantors for the paper.

Funding: The NHS workforce survey was funded under the mental health programme of the NHS executive (Northern and Yorkshire).

Conflict of interest: None.

1 Firth-Cozens J. Emotional distress in junior house officers. BMJ 1987;295:533-6.

2 Ramirez AJ, Graham J, Richards MA, Cull A, Gregory WM. Mental health of hospital consultants: the effects of stress and satisfaction at work. Lancet 1996;347:724-8

3 Hsu K, Marshall V. Prevalence of depression and distress in a large sample of Canadian residents, interns, and fellows. Am J Psych 1987;144:1561-6.

4 Borrill CF, Wall TD, West MA, Hardy GE, Shapiro DA, Carter A, et al. Mental health of the workforce in NHS trusts: phase I final report. Sheffield: Institute of Work Psychology, University of Sheffield, and Leeds: Department of Psychology, University of Leeds, 1996.

5 Goldberg DP. The detection of psychiatric illness by questionnaire. London: Oxford University Press, 1972

(Accepted 5 May 1998)

\title{
The quality and stability of essential drugs in rural Zimbabwe: controlled longitudinal study
}

\author{
Hanif Nazerali, Hans V Hogerzeil
}

Zimbabwe Essential Drugs Action Programme, PO Box CY 924, Harare, Zimbabwe Hanif Nazerali, pharmacist

World Health Organisation, Action Programme on Essential Drugs, 1211 Geneva, Switzerland

Hans V Hogerzeil, medical officer

Correspondence to: Dr Hogerzeil hogerzeilh@who.ch

BMJ 1998;317:512-3
In 1988 an alarming WHO report of substandard ergometrine injection in three developing countries ${ }^{1}$ led to field studies on the stability of essential drugs during international transport to the tropics and to specific stability studies on oxytocic drugs. ${ }^{2}$ We performed a controlled longitudinal study to measure the quality of essential drugs within rural Zimbabwe and to determine whether any failure was due to poor initial quality or to instability of the drugs during inland distribution and storage.

\section{Subjects, methods, and results}

During a two year period (January 1991 to December 1992), samples of drugs arriving at central medical stores in Harare and Bulawayo were retained as controls, and samples of the same batches were later retrieved from hospitals and health centres in five remote, hot, rural districts. We studied 789 samples of 26 brands of 13 essential drugs (selected for relevance to public health and suspected stability problems); 176 samples were taken from central stores and 613 from rural facilities. Of the latter, 261 samples were matched with central samples of the same batch to make longitudinal pairs. Drug quality was measured by level of active ingredient as percentage of stated content and by compliance (pass/fail) with assay standards of the British Pharmacopoeia. Drug stability was measured by comparing mean assay values at central and rural level and by paired analysis of central and rural samples of the same batch.

Drug quality in rural facilities was satisfactory for injectable benzylpenicillin and oral forms of acetylsalicylic acid, amoxicillin, ampicillin, doxycycline, ferrous sulfate, phenylmethoxypenicillin, and tetracycline; it was indeterminate for epinephrine injection. Poor initial quality accounted for problems in injectable ampicillin (2/10 central samples failed, with $87 \%$ and $91 \%$ content) and retinol tablets (5/5 failed; mean assay $73 \%, 95 \%$ confidence interval $63 \%$ to $83 \%$ ), with similar results at rural facilities. An aqueous formulation of injectable procaine benzylpenicillin showed moderate instability with $4 \%(1 \%$ to $6 \%)$ loss after 4.3 months but the assay remained within pharmacopoeial limits. Poor initial quality of all three brands of ergometrine injec-

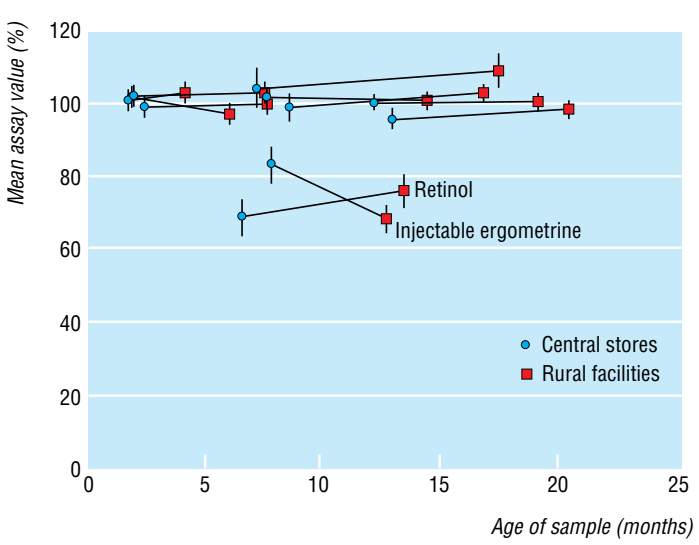

Stability of essential drugs in Zimbabwe. Matched pairs from same batch of retinol (gain) and ergometrine (loss) were unstable acetylsalicylic acid, amoxicillin, ampicillin, benzylpenicillin, doxycycline, epinephrine, ferrous sulfate, phenoxymethylpenicillin, procaine benzylpenicillin, and tetracycline were stable 
tion ( $17 / 26$ failed; mean assay $82 \%, 78 \%$ to $87 \%)$ was compounded by serious instability, with a mean loss of $17 \%$ (13\% to $22 \%)$ after 4.8 months in paired samples. The figure shows the results of the paired analysis. Full data are available on request. ${ }^{3}$

\section{Comment}

Serious instability occurred only with ergometrine injection. This result is in line with two earlier longitudinal studies during transport to and within tropical countries, in which only ergometrine injection, methylergometrine injection, and retinol capsules showed a loss of active ingredient; 12 other essential drugs were stable. ${ }^{45}$ As all three studies focused on essential drugs suspected of being unstable we conclude that, even under the most adverse tropical conditions, clinically relevant instability of essential drugs is rare. Poor initial quality (as with ampicillin, retinol, and ergometrine in our study) poses a much more serious problem as it could, in principle, occur with any drug.

The practical implication of this conclusion is that careful selection of suppliers and quality control at the entry point of the distribution chain are essential to ensure drug quality. Even in tropical climates subsequent quality checks at the district level are not necessary.

Contributors: $\mathrm{HH}$ had the original idea for the study, supervised the design and data analysis, and edited the paper; he will act as guarantor of the study. HN designed the study, collected the samples and the data, performed the data analysis, and wrote the first draft of the paper. Ms Tapuwa Muchemwa coordinated and supervised the laboratory analyses.

Funding: Swedish International Development Authority/ Swedish Agency for Research Cooperation with Developing Countries (SIDA/SAREC) and the WHO Action Programme on Essential Drugs.

Conflict of interest: None.

1 Walker GJA, Hogerzeil HV, Hillgren U. Potency of ergometrine in tropical countries. Lancet 1988;ii:393.

Hogerzeil HV, Walker GJA. Instability of (methyl)ergometrine in tropical climates-an overview. Eur J Obs Gyn Reprod Biol 1996;69:25-9.

3 Nazerali H, Hogerzeil HV. Stability of essential drugs in tropical climates. Inland stability study Zimbabwe. Geneva: World Health Organisation, 1996. (WHO/DAP/94.16.)

4 Hogerzeil HV, De Goeje MJ, Abu Reid IO. Stability of essential drugs in Sudan. Lancet 1991;338:754-5.

5 Hogerzeil HV, Battersby A, Srdanovic V, Stjernstrom NE. Stability of essential drugs during shipment to the tropics. BMJ 1992;304:210-2.

(Accepted 5 May 1998)

\title{
Blood pressure and ageing: longitudinal cohort study
}

\author{
John M Starr, Susan Inch, Susan Cross, William J MacLennan, Ian J Deary
}

Arterial blood pressure rises throughout most of life in industrialised societies. ${ }^{1}$ In old age, however, blood pressure-especially diastolic-falls. ${ }^{1}$ This may be because people who are included in epidemiological studies have diseases or take drugs that affect blood pressure. As greater disease free life expectancy is predicted, the prevalence of hypertension in the elderly may rise if a continued blood pressure increase occurs in those who remain healthy. The few studies examining this provide no consensus. ${ }^{23}$ We investigated whether blood pressure changes in old age relate to health.

\section{Subjects, methods, and results}

We visited 602 (237 men, 365 women) untreated, healthy subjects (mean age 75.7 years, range $70-88$ years) at home. ${ }^{4}$ Educational attainment was recorded and occupation classed as standard occupational classification groups 1-4 (white collar occupations) and groups 5-9 (blue collar occupations). ${ }^{5}$ Blood pressure was measured after the subjects had rested for 25-30 minutes. (A longer version of the methods appears on our web site.)

At follow up (median period 4.20 years, range 3.23-5.23 years), 69 subjects (11.5\%) had died and 105 $(17.4 \%)$ were unavailable. ${ }^{4}$ Health status (documented and self reported) was recorded for the remaining 426 subjects $(70.8 \%)$ and blood pressure remeasured at home. Measurements were of questionable reliability in 41 subjects $(9.6 \%)$ for reasons such as interruptions. Of the remaining 385 subjects (90.4\%), men had significantly lower systolic $(\mathrm{P}=0.01)$ and diastolic $(\mathrm{P}=0.017)$ pressures than women. Occupation significantly influenced diastolic $(\mathrm{P}=0.009)$, but not systolic
$(\mathrm{P}=0.68)$, pressure. Education correlated neither with systolic $(\mathrm{P}=0.44)$ nor diastolic $(\mathrm{P}=0.44)$ pressure. Age correlated negatively with diastolic $(r=-0.124$, $\mathrm{P}=0.01)$, but not with systolic $(r=-0.039, \mathrm{P}=0.2)$, pressure.

At follow up, 195 subjects (50.6\%; 69 men, 126 women) remained healthy and untreated. Correlations between baseline and follow up for systolic and diastolic pressure were 0.49 and 0.24 respectively. When baseline blood pressure was controlled for, neither age nor education significantly affected blood pressure at follow up. When systolic and diastolic pressures were combined in a multivariate repeated measures analysis of variance, sex had no significant effect $(P=0.43)$, but a significant effect was shown for occupation $(\mathrm{P}=0.021)$. A consistent pattern of rises in blood pressure occurred across occupational groups (table) except in groups 8 and 9 . When subjects were stratified by occupation-white collar $(n=119)$ and blue collar $(\mathrm{n}=66)$-a significant rise in blood pressure occurred in white collar $(\mathrm{P}<0.001)$, but not blue collar $(\mathrm{P}=0.08)$, workers.

Incident disease (excluding 29 subjects with hypertension only) was reported or recorded in 163 subjects (42.3\%; 76 men, 87 women). These subjects were older $(\mathrm{P}=0.005)$ than those who remained healthy and more commonly men $(\mathrm{P}=0.021)$, but there were no significant differences in education $(\mathrm{P}=0.40)$ or occupation $(\mathrm{P}=0.067)$. Mean blood pressure remained almost unchanged (table). No significant effects were detected for sex $(\mathrm{P}=0.46)$ or occupation $(\mathrm{P}=0.37)$ over time. Compared with healthy subjects there was a significant change in systolic pressure $(\mathrm{P}=0.003)$ and diastolic pressure $(\mathrm{P}=0.016)$ over time, corrected for age.
Geriatric Medicine Unit, University of Edinburgh EH3 9EW John M Starr, senior lecturer Susan Inch, research nurse Susan Cross, research nurse William J MacLennan, professor

Department of Psychology, University of Edinburgh EH8 9JZ Ian J Deary, professor

Correspondence to: Dr John M Starr, Royal Victoria Hospital, Edinburgh EH4 2DN

John.Starr@ed.ac.uk

BMJ 1998;317:513-4

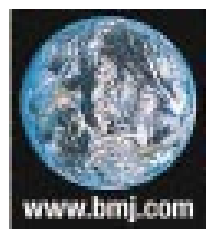

Additional data can be found on our web site 